\title{
Erratum: Hard photoproduction of a diphoton with a large invariant mass [Phys. Rev. D 96, 074008 (2017)]
}

\author{
A. Pedrak, B. Pire, L. Szymanowski, and J. Wagner
}

(Q) (Received 24 July 2019; published 8 August 2019)

We correct the expression for the $\gamma N \rightarrow \gamma \gamma N^{\prime}$ amplitude in the generalized Bjorken regime, where the diphoton is nearly forward and its invariant mass is the hard scale enabling to factorize the scattering amplitude in terms of generalized parton distributions. This amplitude has a very peculiar and interesting analytic structure, while the cross section estimates are only slightly changed.

DOI: $10.1103 /$ PhysRevD.100.039901

Equations (16), (18) of Ref. [1] should read

$A^{V}=2 s\left(V_{k_{1}}-V_{p}+\frac{1+\alpha_{2}}{\alpha_{1}} V_{k 2}\right), \quad B^{V}=2 s\left(-V_{k 2}+V_{p}-\frac{1+\alpha_{1}}{\alpha_{2}} V_{k_{1}}\right), \quad C^{V}=2 s\left(\left(\alpha_{2}-\alpha_{1}\right) V_{p}+V_{k_{2}}-V_{k 1}\right)$,

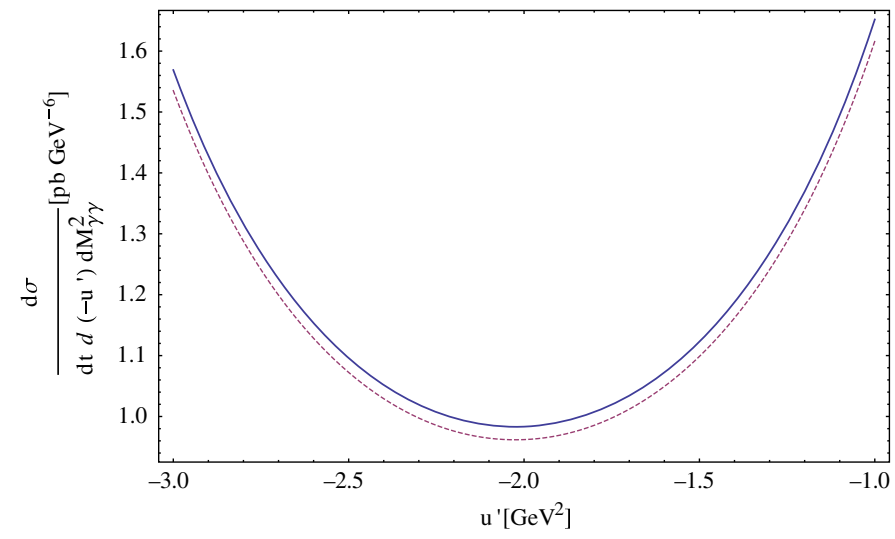

FIG. 2. The $u^{\prime}$ dependence of the unpolarized differential cross section $\frac{d \sigma}{d M_{\gamma \gamma}^{2} d u^{\prime} d t}$ at $t=t_{\min }$ and $S_{\gamma N}=20 \mathrm{GeV}^{2}$, for $M_{\gamma \gamma}^{2}=4 \mathrm{GeV}^{2}$ with the quark GPDs modeled as in [2] (solid curve) and in [3] (dashed curve) for a proton target.
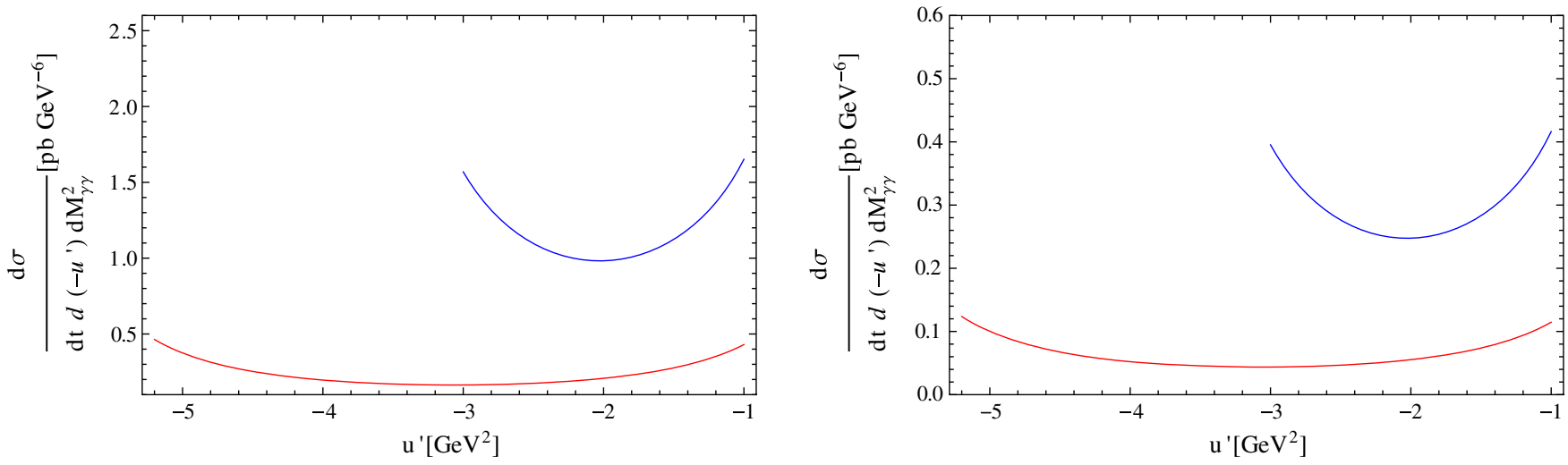

FIG. 3. The $u^{\prime}$ dependence of the unpolarized differential cross section $\frac{d \sigma}{d M_{\gamma \gamma}^{2} d u^{\prime} d t}$ at $t=t_{\min }$ and $S_{\gamma N}=20 \mathrm{GeV}^{2}$, for $M_{\gamma \gamma}^{2}=4 \mathrm{GeV}^{2}$ (blue upper curve) and for $M_{\gamma \gamma}^{2}=6 \mathrm{GeV}^{2}$ (red lower curve), for a proton target (left panel) and a neutron target (right panel). 


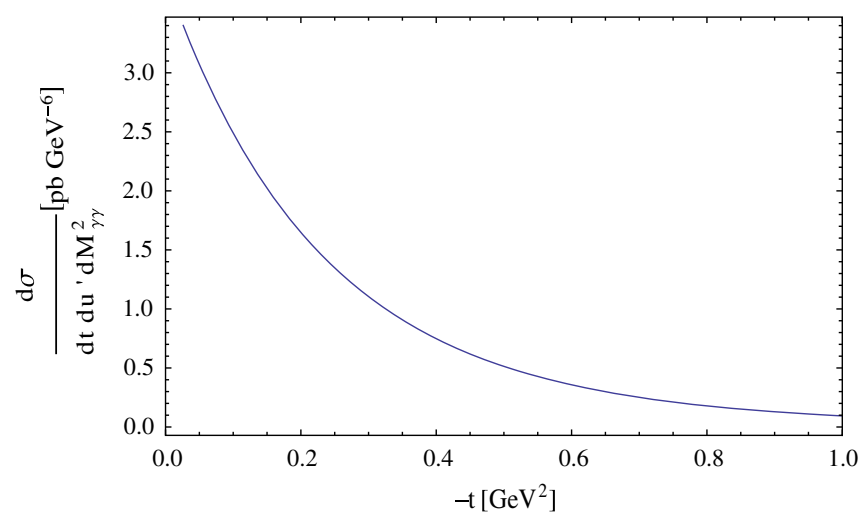

FIG. 4. The $t$-dependence of the unpolarized differential cross section $\frac{d \sigma}{d M_{\gamma \gamma}^{2} d u^{\prime} d t}$ at $u^{\prime}=-2 \mathrm{GeV}^{2}$ and $S_{\gamma N}=20 \mathrm{GeV}^{2}$, for $M_{\gamma \gamma}^{2}=3 \mathrm{GeV}^{2}$.
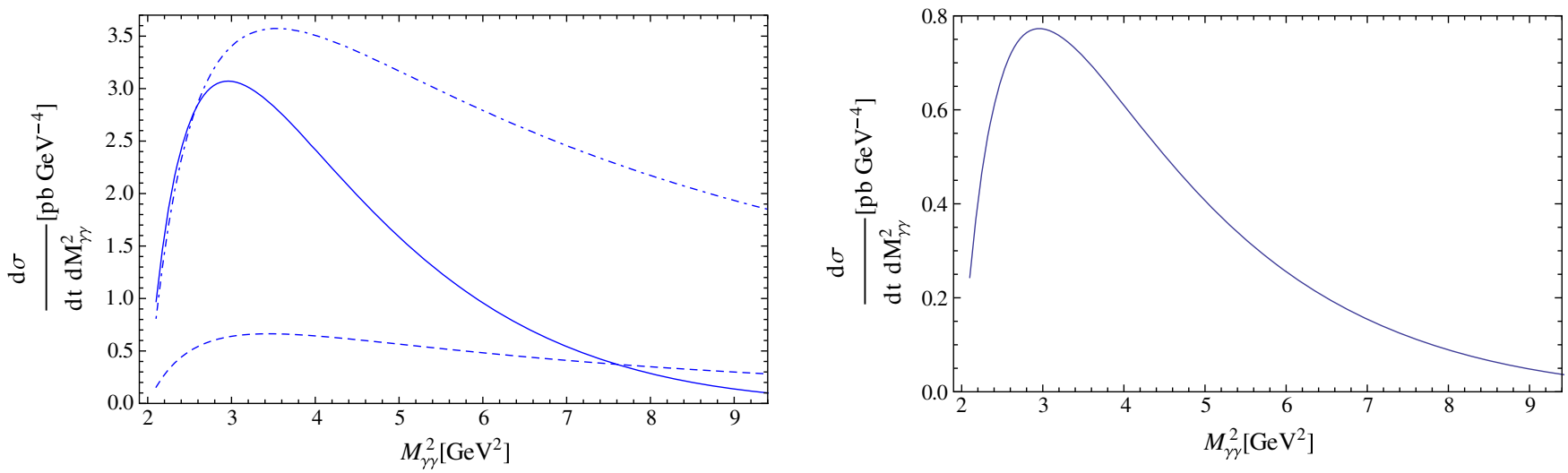

FIG. 5. The $M_{\gamma \gamma}^{2}$ dependence of the unpolarized differential cross section $\frac{d \sigma}{d M_{\gamma \gamma}^{2} d t}$ on a proton (left panel) and on a neutron (right panel) at $t=t_{\min }$ and $S_{\gamma N}=20 \mathrm{GeV}^{2}$ (full curves), $S_{\gamma N}=100 \mathrm{GeV}^{2}$ (dashed curve) and $S_{\gamma N}=10^{6} \mathrm{GeV}^{2}$ (dash-dotted curve, multiplied by $10^{5}$ ).
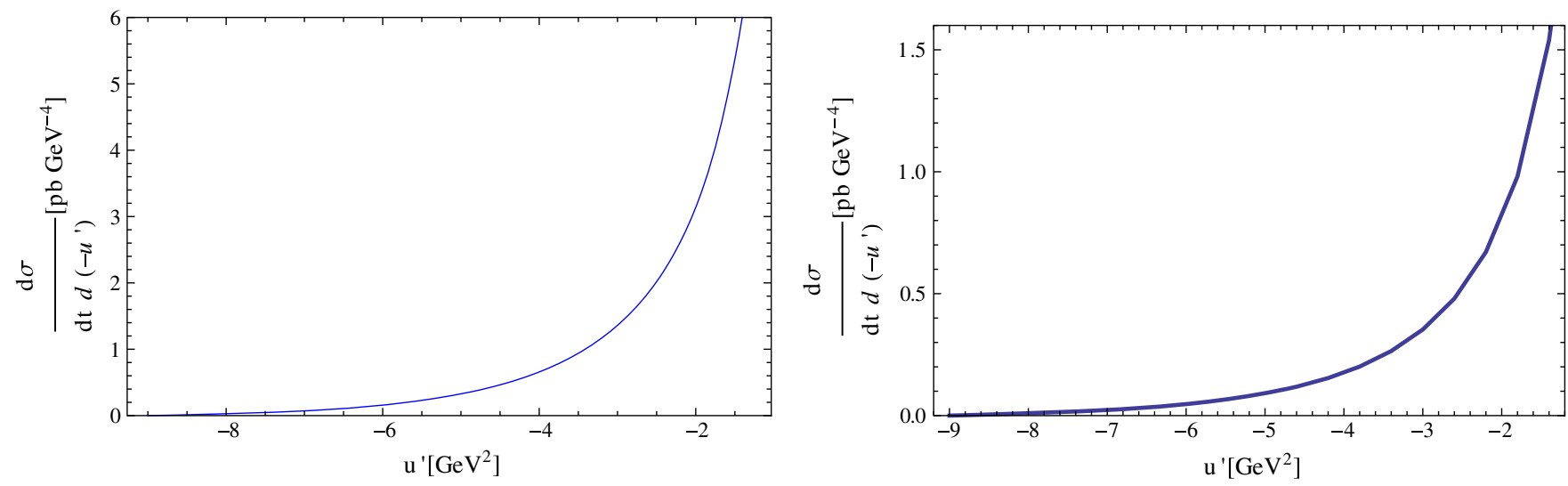

FIG. 6. The $u^{\prime}$ dependence of the unpolarized differential cross section $\frac{d \sigma}{d t d u^{\prime}}$ on a proton (left panel) and on a neutron (right panel) at $t=t_{\min }$ and $S_{\gamma N}=20 \mathrm{GeV}^{2}$, integrated over $M_{\gamma \gamma}^{2}$. 


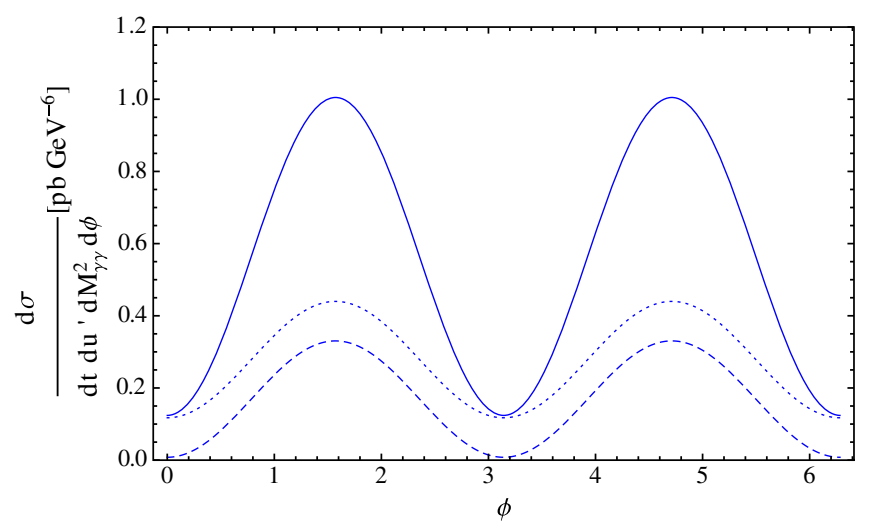

FIG. 7. The azimuthal dependence of the differential cross section $\frac{d \sigma}{d M_{\gamma /}^{2} d t d u^{\prime} d \phi}$ at $t=t_{\min }$ and $S_{\gamma N}=20 \mathrm{GeV}^{2} .\left(M_{\gamma \gamma}^{2}, u^{\prime}\right)=$ $(3,-2) \mathrm{GeV}^{2}$ (solid line), $\left(M_{\gamma \gamma}^{2}, u^{\prime}\right)=(4,-1) \mathrm{GeV}^{2}$ (dotted line), and $\left(M_{\gamma \gamma}^{2}, u^{\prime}\right)=(4,-2) \mathrm{GeV}^{2}$ (dashed line). $\phi$ is the angle between the initial photon polarization and one of the final photon momentum in the transverse plane.

$$
A^{A}=4 i\left(A_{k_{1}}+\frac{1+\alpha_{2}}{\alpha_{1}} A_{k_{2}}-A_{p}\right), \quad B^{A}=4 i\left(-\frac{1+\alpha_{1}}{\alpha_{2}} A_{k_{1}}-A_{k_{2}}+A_{p}\right)
$$

which leads to the vanishing of the real contributions (the principal value integrals) of Eqs. (25)-(27) which sum up to

$$
\begin{aligned}
\mathcal{H}^{q}(\xi) & =\int_{-1}^{1} d x C F_{q}^{V}(x, \xi) H^{q}(x, \xi)=\left(-e_{q}^{3}\right)\left[A^{V} \mathcal{H}_{A^{V}}^{q}(\xi)+B^{V} \mathcal{H}_{B^{V}}^{q}(\xi)+C^{V} \mathcal{H}_{C^{V}}^{q}(\xi)\right], \\
& =\left(-e_{q}^{3}\right)\left(\alpha_{1} A^{V}+\alpha_{2} B^{V}\right) \frac{i \pi}{\xi s^{2} \alpha_{1} \alpha_{2}}\left(H^{q}(\xi, \xi)+H^{q}(-\xi, \xi)\right),
\end{aligned}
$$

and similar equations for $\mathcal{E}^{q}(\xi)$, while Eqs. (28)-(30) remain unchanged.

This leads to the corrected predictions for the cross sections and asymmetries shown in the following figures, which replace Figs. 2-7.

In conclusion, our main conclusions on the feasibility and interest of this reaction are unchanged. The analytic structure of the corrected amplitude is very peculiar and most interesting, while the cross section estimates are only slightly changed.

\section{ACKNOWLEDGMENTS}

We acknowledge a most useful correspondence with Renaud Boussarie.

[1] A. Pedrak, B. Pire, L. Szymanowski, and J. Wagner, Phys. Rev. D 96, 074008 (2017).

[2] S. V. Goloskokov and P. Kroll, Eur. Phys. J. C 53, 367 (2008); P. Kroll, H. Moutarde, and F. Sabatié, Eur. Phys. J. C 73, 2278 (2013).

[3] M. Vanderhaeghen, P. A. M. Guichon, and M. Guidal, Phys. Rev. D 60, 094017 (1999). 